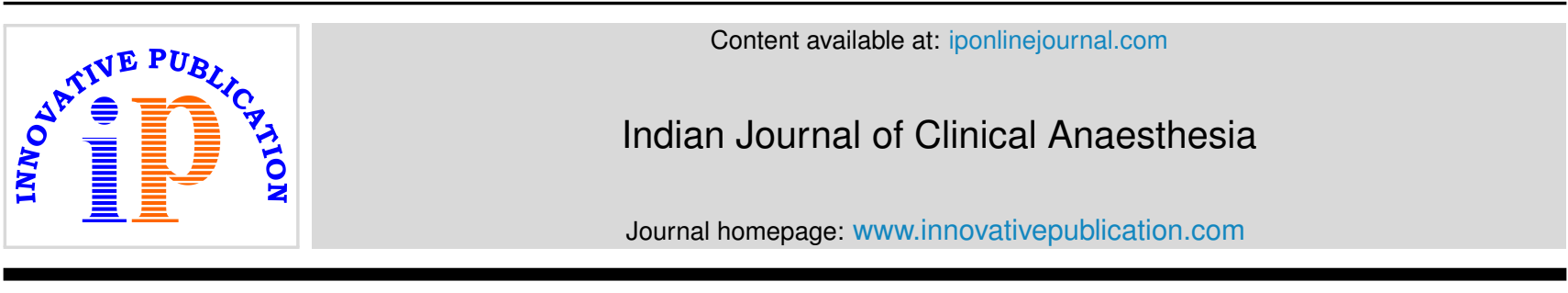

\title{
Editorial
}

\section{Follow the Mantra 'Reduce, Reuse and Recycle' for anaesthetists- to minimize pollution}

\author{
Pallavi Ahluwalia ${ }^{1, *}$ \\ ${ }^{1}$ Teerthanker Mahaveer Medical College and Research Hospital, Moradabad, Uttar Pradesh, India
}

\section{A R T I C L E I N F O}

\section{Article history:}

Received 21-10-2019

Accepted 28-10-2019

Available online 20-09-2019

Keywords:

Pollution

Desflurane.

\begin{abstract}
A B S T R A C T
Few inhalational anaesthetic agents have a global warming potential higher than that of $\mathrm{CO}_{2}$ like Sevoflurane, Isoflurane and Desflurane. Majority of medical nitrous oxide liberates from the use of Entonox, primarily during maternity use. Health professionals have a vital role to play in minimizing and tackling the health impacts and minimizing the climatic change.
\end{abstract}

(C) 2019 Published by Innovative Publication. This is an open access article under the CC BY-NC-ND license (https://creativecommons.org/licenses/by/4.0/)

\section{Introduction}

The introduction of General a naesthesia has enhanced the comfort, outcomes and safety of patients during surgery. The risk reward ratio is high and the benefits of anaesthetic gases are much more as compared to their risks. Recently attention has been drawn to the increase in environmental pollution and climatic change globally because of anaesthetic gases. Eventually all anaesthetic vapors from the circuit go to the environment as they are either exhaled unchanged or are minimally metabolized, hence contributing to anthropogenic climate change. ${ }^{1}$ According to a recent study, the Desflurane concentration is rapi dly increasing in the enviornment. ${ }^{1}$ One bottle of Desflurane produces enough vapors to cause global warming equivalent to $886 \mathrm{~kg} \mathrm{CO}_{2}$.

The absorption characteristic of halogenated agents adds to the greenhouse gas effect by absorbing the infrared radiation that would otherwise have left the Earth's atmosphere. ${ }^{2}$ Few inhalational agents, particularly Isoflurane has ozone depleting effects that can be attributed to interaction of chlorine groups within the molecu le but luckily it has a shorter life span.

\footnotetext{
* Corresponding author.

E-mail address: drpallaviahluwalia@yahoo.com (P. Ahluwalia).
}

Anaesthesia providers must ensure that all anaesthesia equipment's are efficient and maintained to minimize avoidable leaks to minimize atmospheric pollution. The protocol anaesthesia is influenced by various factors such as patient's profile, the type of surgery, and the anaesthetic factors. To promote a safe and a healthy environment, implement simple preventive practices. Behavioral change during administration of anaesthesia can reduce environmental impact. The new er scavenging devices available collect, capture and reuse or even cause destruction of gases, thereby avoiding theater pollution. But in spite of the advantages like safety and benefits of scavenging systems, their usability and reliability, their cost-effectiveness are yet unproven. Xenon, the elemental anaesthetic agent can be substituted but it is too costly, both in terms of finances and emergency intensive distillation from air. To conclude, financial constraints are a major barriers for this sustainable practice.

\section{The strategies to reduce theatre pollution are-}

1. Low flow anaesthesia (LFA) - LFA refers to a technique of using fresh gas flows of $<1$ liter $/ \mathrm{min}$. Feldman has enumerated quite a few recommendations for fresh gas flows to reduce environmental contamination. ${ }^{3}$ Fewer gases like nitrous oxide and Desflurane 
should be used minimally to reduce global warming and ozone depletion.

2. Condensation and destruction of captured vapors - Devices are available that can cause condensation of exhaled anaesthetic vapors from the Scavenging System thereby preventing atmospheric discharge. Theoretically, fractional distillation allows subsequent administration of the separated components but this technique is not yet FDA approved. Most industries use the technology which breaks nitrous oxide to $\mathrm{O}_{2}$ and nitrogen and this is now commonly deployed in small anaesthesia facilities as Excidio (Linde).

3. Total intravenous anaesthesia (TIVA) - Utilising Total intravenous anaesthesia (TIVA) and regional anaesthetic techniques can help minimise the use of volatile anaesthetic agents. Regional anaesthesia techniques combined with general anaesthesia can have 'MAC sparing' effect, by minimising the use of vapour. On one hand TIVA eliminates the greenhouse gas emissions but on the other hand few intravenous drugs exert an environmental impact because of their manufacturing process, transport and syringe driver delivery. The anaesthesia related Green House Gas emissions from Propofol TIVA is 4 times smaller as compared to Desflurane and nitrous oxide.

4. Promoting the use of epidural analgesia or patient controlled analgesiaforlaboring mothers: Can avoid use of ENTONOX (mixture of oxygen and nitrous oxide). Trace amount of Propofol in discarded in syringes is toxic to the aquatic environment. Therefore, it should be discarded in sharp bins for incretion as it requires a temperature of $1000^{\circ} \mathrm{C}$ for its complete destruction. $^{4}$

5. Avoid spillage during filling of a vaporizer and use of fume cupboards for vaporizer filling needs to be promoted. Spilling of inhalational agent can cause significant pollution in the operating theatre. As a safety feature modern vaporizers have special agent-specific filling devices to minimise spillage and pollution.

6. Other measures - Vapor free operation theatre environment can be maintained by venting out the exhaled anaesthetic vapors. Various measures can be employed to minimize exposure such as maintenance of equipment, training the personnel's and monitoring exposure routinely. Now days, AGSS (Anaes thetic Gas Scavenging System) is a legal requirement to minimizes theatre pollut ion. No data is currently available to support association between occupational exposure to anaesthetic agents trace levels and adverse health effects in scavenged areas. Measures to minimize theatre pollution promotes the use of closed or circle systems combined with appropriate fresh gas flows as compared to open or semiclosed systems. Few practical measures such as ensuring good seals during mask ventilation or with airway devices, reducing disconnections between the anaesthetic circuit and machine and use of cuffed tubes in pediatrics can help minimize pollution.

Reducing waste remains the key to improve environmental sustainability during anaesthetic practice and can allow climate change. Anaesthesia generates a large proportion of operating theatre waste. ${ }^{5}$ Reducing waste production is the first step in reducing our environmental impact.

Environmental contamination can be reduced by

1. Reducing the amount of drugs and disposables used and minimizing waste.

2. Purchase of disposable devices made with less material.

3. Reusing as much material as possible.

4. Preference of TIVA over inhalational agents.

5. Avoiding contamination of the environment with drugs that persist, accumulate or are toxic.

6. Maximize recycling possibilities.

7. Having fewer consumables to discard.

8. Ordering less stock so that the stock remains within expiry dates or drugs with long expiry date so that they are not discarded unnecessarily.

9. Opening consumables only when they are needed for example drugs and equipment's.

10. Avoiding unnecessary drug preparation.

11. Prefilled pharmaceutical syringes reduce the amount of pharmaceutical waste produced as they are opened only when they are required.

The most commonly used drug anaesthesia is Propofol. But it has a wastage rate of $32 \%$ out of the total of $45 \%$ of all drugs wasted in a hospital. The reduced size of the drug vials can have a direct impact on the drug wastage.

Another measure to reduce pollution can be to avoid preparation of endotracheal tubes and laryngeal mask airways " just in case it is required". Similarly pre-drawing up medications can be avoided. A voiding frequent change of anaesthetic machine tubing can help minimise waste.

The policy of 'reduce, reuse, recycle' can help combat pollution and save the environment.

\section{References}

1. Vollmer M, Rhee T, Rigby M. Modern inhalation anesthetics: potent greenhouse gases in the global atmosphere. Geophys Res Lett. 2015;42:1606-1611.

2. Andersen MPS, Nielsen OJ, Wallington TJ, Karpichev B, Sander SP. Medical intelligence article: assessing the impact on global climate from general anesthetic gases. Anesth Analg. 2012;114(5):1081-1085.

3. Managing fresh gas flow to reduce environmental contamination. Anesth Analg. 2012;114:1093-1101.

4. Mankes RF. Propofol wastage in anesthesia. Anesth Analg. 2012;114:1091-1093.

5. Pungsornruk K, Forbes MP, Hellier C, Bryant M. A renewed call for environmentally responsible anaesthesia. Anaesth Intensive Care. 2015;43(6):800-801. 


\section{Author biography}

Pallavi Ahluwalia Professor
Cite this article: Ahluwalia P. Follow the Mantra 'Reduce, Reuse and Recycle' for anaesthetists- to minimize pollution. Indian J Clin Anaesth 2019;6(4):478-480. 